\title{
Terminación de la psicoterapia desde la perspectiva de los pacientes
}

\section{Psychotherapy Termination from the Patients' Perspective}

\author{
Daniel Espinosa-Duque 1 ORCID , David Alejandro Ibarra ${ }^{2} \underline{\mathrm{ORCID}}$, Diana María Ocampo Lopera ${ }^{3}$ \\ $\underline{O R C I D}$, Linda Montoya ${ }^{4} \underline{O R C I D}$, Adriana Hoyos-Pérez ${ }^{5} \underline{0 R C I D}$, Luisa Fernanda Medina ${ }^{6} \underline{O R C I D}$, Santiago \\ Tamayo $^{7} \underline{\mathrm{ORCID}}$, Julieth Zapata Restrepo ${ }^{8} \underline{\mathrm{ORCID}}$
}

${ }^{1}$ Instituto Milenio para la Investigación de la Depresión y la Personalidad (MIDAP),

Chile

1357 Universidad CES

2468 Universidad de San Buenaventura

Colombia

Fecha correspondencia:

Recibido: diciembre 18 de 2019.

Aceptado: noviembre 13 de 2020.

Forma de citar:

Espinosa-Duque, D., Ibarra, D.A., Ocampo Lopera, D.M. Montoya,

L., Hoyos-Pérez, A., Medina, L.F., Tamayo, S., \& Zapata Restrepo,

J. (2020). Terminación de la psicoterapia desde la perspectiva de los pacientes Rev. CES PSico, 13(3), 201-221.

$\frac{\text { Open access }}{\text { (c) Copyright }}$

Licencia creative commons

Etica de publicaciones

Revisión por pares

Gestión por Open Journal System DOl: http://dx.doi.org/10.21615/ cesp. 13.3.12

ISSN: 2011-3080

Comparte

\section{Resumen}

El aumento de la oferta de servicios de psicoterapia en las últimas décadas justifica el estudio de los elementos relacionados con su terminación, y su relación con el cumplimiento de los objetivos y la adherencia de los pacientes al tratamiento. Objetivo: identificar los factores que influyen en la terminación de los procesos psicoterapéuticos desde la perspectiva de los pacientes. Metodología: se utilizó un diseño de investigación mixto de triangulación concurrente; se aplicaron encuestas estructuradas y entrevistas en profundidad sobre el fin de la terapia. A nivel cuantitativo se realizaron análisis descriptivos y de regresión, y, a nivel cualitativo, reducción de datos, comparación constante y triangulación de la información. Participaron 137 pacientes de dos centros universitarios de atención psicológica en Antioquia (Colombia), 100 participantes respondieron una encuesta y los restantes una entrevista cualitativa. Resultados: se identificaron tres dimensiones relacionadas con la terminación de los procesos psicoterapéuticos: el cumplimiento o no de los objetivos, el tipo de atribución causal del paciente (interna o externa) y la persona que decide finalizar. La mayoría de los pacientes del estudio dejaron de asistir a psicoterapia sin cumplir sus objetivos o con un cumplimiento parcial de estos, por atribuciones externas y por decisión propia. Discusión: los aspectos institucionales, terapéuticos y de la vida del paciente tienen un rol significativo en la terminación de los procesos psicoterapéuticos. Además, se plantea la importancia de considerar en la formación de los terapeutas las características de los procesos de cierre y los diferentes marcadores asociados con la terminación prematura.

Palabras clave: Psicoterapia, Fin de Psicoterapia, Terminación de Psicoterapia, Abandono, Cierre Terapéutico, Adherencia, Alianza Terapéutica, Psicología Clínica. 
Sobre los autores:

1. Doctor en Psicoterapia.

Docente Investigador

Universidad CES.

Instituto Milenio para la

Investigación en Depresión

y Personalidad, MIDAP.

2. Magíster. Docente Investigador Universidad de San Buenaventura, Medellín, Colombia.

3. Magister. Docente Investigadora Universidad CES. Coordinadora Centro de Atención en Salud, Universidad CES.

4. Psicóloga. Egresada Universidad USB-Medellín.

5. Psicóloga. Egresada Universidad CES.

6. Psicóloga. Egresada Universidad USB-Medellín.

7. Psicóloga. Egresada Universidad CES.

8. Magíster. Docente Investigadora Universidad de San Buenaventura, Medellín, Colombia.

\section{Abstract}

The increase in the supply of psychotherapy services in recent decades justifies the study of the elements related to its termination. This is one of the main topics to understand achievement of therapeutic goals and adherence of patients in psychotherapy. Aim: to identify the factors that influence termination of psychotherapeutic processes from the patients' perspective. Method: a mixed research design of concurrent triangulation was used; and structured surveys inquiring and in-depth interviews about psychotherapy termination were conducted. It was carried out at the quantitative level, both descriptive and regression analyses, and at the qualitative level, data reduction, constant comparison, and triangulation of information. A total of 137 patients from two higher education centers of psychological care in Antioquia (Colombia) participated, 100 answered a survey and the remaining participants were interviewed in-depth. Results: Results show three dimensions that allow various conceptualizations of therapy termination: achievement of goals, causal attribution and the person who decides to terminate. Most of the patients in the study dropped out of psychotherapy without the completion of their objectives or with a partial completion of these, due to external attributions and their own decision. Discussion: it was discussed how institutional, therapeutic, and life patient's aspects have a significant role in termination of psychotherapeutic processes. As well as the importance of considering therapists' training in relation to the characteristics of these processes and the different indicators associated with premature termination.

Keywords: Psychotherapy, Psycotherapy Termination, Psychotherapy Dropout, Adherence, Therapeutic Alliance, Clinical Psychology, Mental Health.

\section{Introducción}

Los tratamientos psicoterapéuticos en Colombia han tenido un significativo crecimiento en las últimas décadas (Rojas-Bernal, Castaño-Pérez, \& Restrepo-Bernal, 2018; Campo-Arias, Oviedo, \& Herazo, 2014), como parte de los servicios del sistema de salud pública (MINSALUD, 2018) y la atención en instituciones privadas. En este contexto, la calidad en la prestación de los servicios y sus características son de gran interés para los profesionales y las instituciones de salud mental (González et al., 2016). Con el fin de brindar atención más oportuna y pertinente a los pacientes, algunas instituciones, profesionales e investigadores en el área de la salud mental se han preguntado por los factores que inciden en el acceso, permanencia y efectividad de los procesos terapéuticos (Lambert, 2012a), así como en las razones de su terminación y los factores relacionados (Rondón, Otálora, \& Salamanca, 2009).

La mayoría de los estudios que hacen referencia a la terminación de la psicoterapia se focalizan en una de sus formas, conceptualizada como abandono o deserción (drop-out) (Swift \& Greenberg, 2014; Sharf, Primavera, \& Diener, 2010; Gersh et al., 2017). Otros autores, han señalado la importancia de conocer las razones generales por las que finaliza la psicoterapia y las variables asociadas (Jaramillo et al., 2018); lo que permite ampliar la comprensión de la relación de los tipos de terminación con otros elementos como las dificultades institucionales, características del terapeuta o del ambiente social (Rondón et al., 2009).

La terminación o fin del proceso se da cuando después de un encuentro y proceso formal en el espacio psicoterapéutico, se genera un cierre o corte entre el consultante y el psicoterapeuta o institución (Swift \& Greenberg, 2012; Vélez \& Restrepo, 2008); que puede clasificarse en tres tipos: abandono, acordada mutuamente y cierre realizado por el terapeuta. 
Pág 203

Algunos estudios a nivel mundial informan una alta tasa de abandono, que se sitúa entre el 20 y $60 \%$ (Berghofer, Schmidl, Rudas, Steiner, \& Schmitz, 2002; Swift \& Greenberg, 2012; Wierzbicki \& Pekarik, 1993); y en el ámbito latinoamericano reportan porcentajes entre el 38 y el 63 \% (De la Parra, Gómez-Barris, Zúñiga, Dagnino, \& Valdés, 2018; Jo \& Almao, 2002).
El primer tipo, el abandono se define como la terminación de una intervención psicoterapéutica por decisión del consultante, en un momento en el que no se han alcanzado los objetivos de la terapia o finalizado la intervención. Algunos estudios a nivel mundial informan una alta tasa de abandono, que se sitúa entre el 20 y $60 \%$ (Berghofer, Schmidl, Rudas, Steiner, \& Schmitz, 2002; Swift \& Greenberg, 2012; Wierzbicki \& Pekarik, 1993); y en el ámbito latinoamericano reportan porcentajes entre el 38 y el 63 \% (De la Parra, Gómez-Barris, Zúñiga, Dagnino, \& Valdés, 2018; Jo \& Almao, 2002).

Algunas características del paciente y factores asociados con el abandono son: adicciones (Rondón et al., 2009), trastornos de personalidad (Persons, Burns, \& Perloff, 1988), creencias frente a la enfermedad (Berghofer et al., 2002; Rondón et al., 2009), bajo nivel de formación (Swift \& Greenberg, 2012), estigma asociado a la terapia y la enfermedad mental (Borghi, 1968; Lambert, 2012b; Rondón et al., 2009), y expectativas y creencias sobre la terapia (Borghi, 1968; Hopwood, Ambwani, \& Morey, 2007; Santibáñez et al., 2008; Moreno, Rozo, \& Cantor, 2012).

Asimismo, se identifican factores sociodemográficos relacionados con la deserción o abandono terapéutico como: un nivel socioeconómico bajo (Barrett, Chua, Crits-Christoph, Gibbons, \& Thompson, 2008; Lambert, 2012a; Reis \& Brown, 1999; Rondón et al., 2009), angustia financiera (Thompson, Goldberg, \& Nielsen, 2018), bajo nivel educativo (Baekeland \& Lundwall, 1975; Barrett et al., 2008; Lambert, 2012a), pertenecer a una minoría étnica (Baekeland \& Lundwall, 1975; Lambert, 2012a; Reis \& Brown, 1999) o a grupos etarios extremos (Baekeland \& Lundwall, 1975; Barrett et al., 2008; Rondón et al., 2009).

Otros aspectos relacionados con el abandono tienen que ver con algunas condiciones de acceso a la institución y características propias del tratamiento, entre ellas: la distancia y tiempo de desplazamiento al lugar de atención (Rondón et al., 2009; Seclén-Palacín \& Darras, 2005); la consecución de citas (Hopwood et al., 2007); el acuerdo (o no) frente a las expectativas que posee el paciente y las posibilidades que puede ofrecer la terapia (Hopwood et al., 2007; Reis \& Brown, 1999) y, el acceso, continuidad y elementos contextuales del servicio (Moreno et al, 2012, Westmacott, Hunsley, Best, Rumstein-McKean, \& Shindler, 2010). Al poner el foco en la relación con el terapeuta, se encuentra que los pacientes desertan cuando perciben falta de experiencia en el profesional (Baekeland \& Lundwall, 1975; Hunsley, Aubry, Verstervelt, \& Vito, 1999) o no se genera la necesidad de terapia durante las primeras sesiones (Fernández-Arias, García-Fernández, \& Bernaldo-de-Quirós, 2016).

El segundo tipo, la terminación acordada mutuamente se entiende como el arreglo entre psicoterapeuta y paciente de no continuar un proceso porque se han alcanzado los objetivos (Westmacott et al., 2010), o porque se identifica en las condiciones del paciente, del entorno o de la terapia, alguna dificultad para el desarrollo del tratamiento (Tjeltveit, 2006; Rancich, Pérez, Gelpi, \& Mainetti, 1999). Estos factores que pueden generar una terminación por acuerdo mutuo sin cumplimiento de objetivos, pueden ser, por ejemplo, dificultades horarias o de recursos económicos (Rondón et al., 2009; Westmacott et al., 2010), o preocupaciones identificadas por alguno de los participantes (Vélez \& Restrepo, 2008).

El tercer tipo, es la terminación propuesta por el terapeuta que puede relacionarse con características del paciente, cuando este no cumple con el encuadre mínimo acordado para recibir la atención (ej. asistencia, puntualidad, indicaciones, etc.), con 
El presente estudio tiene como objetivo identificar los factores que influyen en la finalización de los procesos psicoterapéuticos desde la perspectiva de los pacientes de dos centros universitarios de atención psicológica en Antioquia (Colombia). elementos de la institución, cuando los lineamientos y aspectos logísticos afectan ( $p$. ej. políticas de cancelación de citas) (Swift \& Greenberg, 2014) y con aspectos personales o laborales del terapeuta (p. ej. rotación del personal, momentos de crisis, etc.) (De la Parra, Errázuriz, Gómez-Barris, \& Zúñiga, 2019; Rondón et al., 2009; Hunsley et al., 1999). Una contratransferencia negativa también puede tener un rol importante en la decisión del psicoterapeuta de terminar unilateralmente un proceso o remitir un paciente (Fair \& Bressler, 1992).

En Colombia, algunos centros de atención psicológica han realizado investigaciones sobre los motivos de permanencia y deserción de los pacientes (Rondón et al., 2009); y han encontrado que la permanencia no suele ser tan larga como tradicionalmente se piensa, dado que en la séptima sesión $50 \%$ de los pacientes ha dejado de acudir y en promedio asisten a 11,17 sesiones (Jaramillo et al., 2018). En la misma dirección, estudios a nivel internacional han encontrado que los pacientes asisten a cinco o seis sesiones en promedio (Lambert, 2012a). Además, se ha evidenciado un alto porcentaje de procesos psicoterapéuticos sin reportes respecto a los motivos de terminación en el registro de historias clínicas (Jaramillo et al., 2018); situación que revela la necesidad de estudiar aquellos factores asociados a la finalización de los procesos terapéuticos.

La comprensión de los factores asociados a la terminación de la terapia puede redundar en beneficio tanto de los profesionales y la academia, como de los pacientes, quienes finalmente dan cuenta del sentido y calidad del trabajo terapéutico (Fernández-Álvarez \& Castonguay, 2018). Además, contemplar la perspectiva de los pacientes, permite reflexionar sobre los modelos de atención, optimizar los servicios que se ofrecen, brindar una intervención más cercana a sus características y contextos de atención (De la Parra et al., 2018), y proporcionar elementos fundamentales para la formación de psicoterapeutas.

De acuerdo con lo anteriormente expuesto, el presente estudio tiene como objetivo identificar los factores que influyen en la finalización de los procesos psicoterapéuticos desde la perspectiva de los pacientes de dos centros universitarios de atención psicológica en Antioquia (Colombia).

\section{Metodología}

El presente estudio tiene un diseño mixto de triangulación concurrente (Hernández-Sampieri \& Mendoza, 2018), mediante el cual se busca realizar la validación entre datos cualitativos y cuantitativos, con el objetivo de establecer, a través de información complementaria, la confirmación de los resultados previos sobre un mismo fenómeno. Por lo tanto, se recolectaron datos sobre los factores que influyen en la terminación de los procesos psicoterapéuticos en instituciones desde la perspectiva de los pacientes; inicialmente, se adelantó un análisis independiente y, luego, un proceso de comparación y análisis conjunto, que permitió observar los niveles de convergencia de los resultados (Creswell \& Plano, 2007).

\section{Participantes}

La investigación se llevó a cabo en dos centros de atención psicológica, la IPS CES Sabaneta de la Universidad CES (en adelante CES) y el Consultorio Psicológico Popular CPP de la Universidad de San Buenaventura (en adelante USB), durante el año 2019. Los criterios de inclusión de los participantes fueron: haber estado en psicoterapia en uno de los centros de la investigación, tener 18 años o más, y aceptar participar en el estudio mediante consentimiento informado. Fueron excluidos de la 
investigación los pacientes con daño orgánico cerebral, retardo mental y que hubieran experimentado un episodio psicótico en el último año. Los participantes fueron divididos en dos grupos, según la forma de recolección de los datos y el tipo de análisis realizado:

1. Análisis cuantitativo: en este nivel, se tomaron como punto de partida los Registros Individuales de Prestación de Servicios de Salud (RIPS), luego se filtraron los pacientes que cumplieran los criterios de inclusión y se realizó un muestreo aleatorio estratificado por rangos de edad. De los pacientes identificados, $38 \%$ no pudieron ser contactados, $12 \%$ no cumplieron con la verificación de los criterios del estudio, $18 \%$ no aceptaron participar y $30 \%$ aceptaron. Finalmente, participaron 100 pacientes que habían asistido al servicio de psicoterapia y no reportaban asistencia en los últimos cuatro meses (50 en cada institución); quienes contestaron una encuesta. Este grupo estuvo conformado por 68 mujeres y 32 hombres, entre 18 y 80 años ( $X$ de edad $=40,18 ; \mathrm{DE}=17$ ).

2. Análisis cualitativo: a partir de un muestreo intencional se seleccionaron 37 pacientes de las mismas instituciones, con quienes se realizó una entrevista cualitativa semiestructurada sobre la experiencia en psicoterapia. El tamaño de la muestra en este subgrupo fue determinado por el criterio de estabilización de categorías. Estos pacientes también habían estado en un proceso psicológico anteriormente y no tenían sesiones programadas en el momento de la entrevista. Este grupo estuvo conformado por 12 hombres y 25 mujeres (CES: 3 hombres y 15 mujeres / USB: 9 hombres y 10 mujeres) entre 22 y 77 años.

\section{Factores, instrumentos y recolección de datos}

Para ambos tipos de análisis se llevó a cabo un proceso de recolección de información que permitiera dar cuenta de los elementos que influyen en la finalización de los procesos psicoterapéuticos.

1. Análisis cuantitativo: en este nivel, se realizó una encuesta con diferentes tipos de preguntas (tipo Likert, dicotómicas y preguntas abiertas) sobre datos sociodemográficos, razones del fin de terapia, factores socioeconómicos, factores institucionales, motivación para realizar la terapia, valoración de las acciones terapéuticas, vínculo terapéutico, progreso terapéutico, y satisfacción con la terapia y la institución. Dado que no fue identificado un instrumento que indagara específicamente sobre el tema, se construyó una encuesta con un grupo de expertos para determinar los dominios de análisis, y se hizo una triangulación con elementos del rastreo teórico para determinar la construcción de los reactivos. Las encuestas se realizaron vía telefónica, se audio-grabaron y registraron en una planilla para su posterior análisis. Este análisis se realizó con las variables que se relacionan en la Tabla 1 
Tabla. 1. Variables incluidas en los análisis cuantitativos

\begin{tabular}{|c|c|c|c|}
\hline Grupo & Variables & Valores & $\begin{array}{l}\text { Tipo de } \\
\text { Variable }\end{array}$ \\
\hline \multirow{8}{*}{$\begin{array}{c}\text { Variables } \\
\text { dependientes: } \\
\text { Razones de } \\
\text { terminación de la } \\
\text { terapia }\end{array}$} & $\begin{array}{l}\text { Por aspectos propios del } \\
\text { paciente }\end{array}$ & Ausente - Presente & Dicotómica \\
\hline & $\begin{array}{c}\text { Por aspectos del paciente } \\
\text { externos a él }\end{array}$ & Ausente - Presente & Dicotómica \\
\hline & $\begin{array}{l}\text { Por aspectos asociados a la } \\
\text { institución o sistema de salud }\end{array}$ & Ausente - Presente & Dicotómica \\
\hline & $\begin{array}{c}\text { Por cumplimiento de } \\
\text { expectativas del paciente }\end{array}$ & Ausente - Presente & Dicotómica \\
\hline & Cierre por parte del terapeuta & Ausente - Presente & Dicotómica \\
\hline & $\begin{array}{l}\text { Por cumplimiento de tareas o } \\
\text { metas }\end{array}$ & Ausente - Presente & Dicotómica \\
\hline & $\begin{array}{l}\text { Asociada a aspectos } \\
\text { socioeconómicos }\end{array}$ & Ausente-Presente & Dicotómica \\
\hline & $\begin{array}{l}\text { Asociada a aspectos } \\
\text { institucionales }\end{array}$ & Ausente - Presente & Dicotómica \\
\hline \multirow{9}{*}{$\begin{array}{c}\text { Variables } \\
\text { independientes: } \\
\text { Sociodemográficas } \\
\text { del paciente }\end{array}$} & Edad & Años & Cuantitativa \\
\hline & Género & Masculino - Femenino & Dicotómica \\
\hline & Estrato & $1-2-3-4-5-6$ & Escalar \\
\hline & Ocupación & $\begin{array}{c}\text { Estudia/trabaja - No } \\
\text { Estudia / trabaja }\end{array}$ & Dicotómica \\
\hline & \multirow{4}{*}{ Vive con: } & Solo: Si - No & Dicotómica \\
\hline & & Padres: Si - No & Dicotómica \\
\hline & & Hijos: Si - No & Dicotómica \\
\hline & & Pareja: Si - No & Dicotómica \\
\hline & Nivel de formación & $\begin{array}{l}\text { Niveles de estudio } \\
\text { cursados }\end{array}$ & Escalar \\
\hline \multirow{4}{*}{$\begin{array}{c}\text { Variables } \\
\text { independientes: de } \\
\text { la terapia }\end{array}$} & $\begin{array}{c}\text { Nivel de formación del } \\
\text { terapeuta }\end{array}$ & Posgrado - pregrado & Dicotómica \\
\hline & Tipo de diagnóstico & $\begin{array}{c}\text { Trastornos mentales } \\
\text { y de la conducta - } \\
\text { Problemáticas de la } \\
\text { cotidianidad }\end{array}$ & Dicotómica \\
\hline & Sesiones asistidas & Número & Escalar \\
\hline & Tipo de motivo de Consulta & $\begin{array}{l}\text { Problemáticas de } \\
\text { la cotidianidad } \\
\text { o relacionales } \\
\text { - Dificultades } \\
\text { psicosomáticas o del } \\
\text { estado del ánimo }\end{array}$ & Dicotómica \\
\hline \multirow{6}{*}{$\begin{array}{c}\text { Variables } \\
\text { independientes: } \\
\text { Percepción del } \\
\text { paciente de la } \\
\text { terapia }\end{array}$} & Motivación & $1-2-3-4-5$ & Escalar \\
\hline & $\begin{array}{l}\text { Acciones } \\
\text { terapéuticas }\end{array}$ & $1-2-3-4-5$ & Escalar \\
\hline & $\begin{array}{c}\text { Vínculo } \\
\text { terapéutico }\end{array}$ & $1-2-3-4-5$ & Escalar \\
\hline & $\begin{array}{l}\text { Progreso } \\
\text { terapéutico }\end{array}$ & $1-2-3-4-5$ & Escalar \\
\hline & Recomendaría & $1-2-3-4-5$ & Escalar \\
\hline & Nivel de Satisfacción & $1-2-3-4-5$ & Escalar \\
\hline
\end{tabular}


Pág 207

Con los datos cuantitativos se llevó a cabo un proceso de organización y estandarización de las respuestas abiertas y se realizaron los análisis estadísticos, y con los cualitativos se realizó la lectura analítica y construcción de categorías. Finalmente, se efectuaron los procesos de integración de los diferentes tipos de análisis.
2. Análisis cualitativo: Se realizaron entrevistas cualitativas semiestructuradas, con el objetivo de explorar la experiencia subjetiva de los participantes en algunos tópicos de la psicoterapia (Kvale \& Brinkmann, 2009; Larsen, Flesaker, \& Stege, 2008), tales como, las razones para su terminación. Las entrevistas se adelantaron cara a cara, se audio-grabaron y transcribieron para su posterior análisis. Solo en casos excepcionales se consideró realizarlas telefónicamente o a través de otro medio de comunicación a distancia.

\section{Procedimiento}

Con la respectiva autorización de los centros de atención psicológica, en primer lugar, se revisaron los RIPS y se seleccionaron los pacientes que cumplieran con los criterios de inclusión. Posteriormente, fueron invitados a participar en el estudio y, una vez leídos el consentimiento y las normas éticas, si aceptaban participar, se procedió con la recolección, transcripción y sistematización de los datos. Con los datos cuantitativos se llevó a cabo un proceso de organización y estandarización de las respuestas abiertas y se realizaron los análisis estadísticos, y con los cualitativos se realizó la lectura analítica y construcción de categorías. Finalmente, se efectuaron los procesos de integración de los diferentes tipos de análisis.

\section{Análisis de datos}

Como es usual en este tipo de diseños, los análisis cuantitativos y cualitativos se realizaron de manera independiente $y$, posteriormente, se identificaron las convergencias y divergencias de los datos mediante el proceso de triangulación.

Análisis cuantitativo: se calcularon los estadísticos descriptivos de las respuestas de las encuestas, se consideraron las frecuencias, porcentajes, promedios y desviaciones estándar, según el tipo de variable. Posteriormente, se realizaron regresiones logísticas para identificar las variables independientes asociadas con las razones de terminación de terapia por medio del software SPSS. Como se puede observar en la Tabla 1, las variables independientes incluidas en el modelo de análisis corresponden a características sociodemográficas de la terapia y del paciente y, las dependientes, a la presencia o ausencia de determinadas razones de terminación de la terapia.

Análisis cualitativo: se adelantó a partir de la reducción de datos (Rodríguez, Lorenzo, \& Herrera, 2005), proceso en espiral que implica la integración constante de los datos obtenidos (Mejía \& Manjarrés, 2011). En el proceso de codificación, comparación constante y categorización (Monge, 2015), emergieron 11 códigos: logro, expectativa, valoración del proceso, percepción de la intervención, factores institucionales, inconformidad, límites, motivación, cierre, inconvenientes del cierre, factores económicos; y tres categorías: incumplimiento de objetivos, factores institucionales y logro de objetivos. Para realizar este procedimiento se utilizaron procesadores de texto.

\section{Resultados}

\section{Cuantitativos}

Los 100 participantes encuestados refirieron 125 razones para terminar la psicoterapia, que fueron agrupadas en 19 subtipos y en cinco grupos, según el tipo de atribución (externa institucional, externa del paciente o interna) y el cumplimiento de tareas y objetivos (prematuro o con cumplimiento). 
En primer lugar, las terminaciones prematuras se subdividieron así: asociadas a elementos de la institución de salud ( $F=39)$, relacionadas con aspectos del paciente, y externas a él $(F=34)$, y referidas a aspectos propios del paciente $(F=29)$. En segunda instancia, cuando la terapia terminó con cumplimiento de objetivos o tareas se agruparon así: cierre por parte del terapeuta $(F=5)$, remisiones o derivaciones a otros profesionales $(F=2)$ o terminación por parte del paciente por cumplimiento de expectativas $(F=17)$. Estos resultados se observan de manera específica en la Tabla 2.

Tabla 2. Razones para la terminación de la psicoterapia reportada por los pacientes

\begin{tabular}{|c|c|c|c|c|c|c|}
\hline $\begin{array}{c}\text { Tipo de } \\
\text { atribución }\end{array}$ & Término $P$ & rematuro & $\boldsymbol{F}$ & $\begin{array}{c}\text { Término con } \\
\text { cumplimiento } \\
\text { de objetivos o } \\
\text { tareas }\end{array}$ & & $F$ \\
\hline \multirow{12}{*}{$\begin{array}{c}\text { Atribución } \\
\text { externa al } \\
\text { paciente }\end{array}$} & \multirow{8}{*}{$\begin{array}{c}1 . \\
\text { Correspondientes } \\
\text { a la institución o } \\
\text { sistema de salud } \\
\text { (F: 39) }\end{array}$} & $\begin{array}{l}\text {-Ubicación del } \\
\text { centro }\end{array}$ & 13 & \multirow{12}{*}{$\begin{array}{l}\text { 4. Cierre de la } \\
\text { psicoterapia } \\
\text { por parte del } \\
\text { terapeuta } \\
\text { (F: 7) }\end{array}$} & $\begin{array}{c}\text {-Cumplimiento } \\
\text { de Objetivos }\end{array}$ & 5 \\
\hline & & $\begin{array}{l}\text {-Cambio de } \\
\text { terapeuta }\end{array}$ & 7 & & \multirow{15}{*}{-Remisión } & \multirow{11}{*}{2} \\
\hline & & $\begin{array}{c}\text {-Finalización de } \\
\text { citas }\end{array}$ & 5 & & & \\
\hline & & $\begin{array}{c}\text {-Limitaciones } \\
\text { horarias }\end{array}$ & 5 & & & \\
\hline & & $\begin{array}{c}\text {-Dificultades con } \\
\text { el terapeuta }\end{array}$ & 4 & & & \\
\hline & & $\begin{array}{c}\text {-Aspectos } \\
\text { institucionales }\end{array}$ & 3 & & & \\
\hline & & $\begin{array}{l}\text {-Información } \\
\text { sobre la terapia }\end{array}$ & 3 & & & \\
\hline & & $\begin{array}{l}\text {-Sistema de } \\
\text { salud }\end{array}$ & 3 & & & \\
\hline & \multirow{4}{*}{$\begin{array}{c}2 . \\
\text { Correspondientes } \\
\text { a aspectos } \\
\text { paciente externos } \\
\text { a él } \\
(F: 34)\end{array}$} & $\begin{array}{l}\text {-Tiempo del } \\
\text { paciente }\end{array}$ & 22 & & & \\
\hline & & $\begin{array}{l}\text {-Dificultades } \\
\text { económicas }\end{array}$ & 9 & & & \\
\hline & & $\begin{array}{c}\text {-Problemas } \\
\text { familiares }\end{array}$ & 3 & & & \\
\hline & & $\begin{array}{c}\text {-Condiciones de } \\
\text { salud }\end{array}$ & 2 & & & \\
\hline \multirow{4}{*}{$\begin{array}{c}\text { Atribución } \\
\text { interna del } \\
\text { paciente }\end{array}$} & \multirow{4}{*}{$\begin{array}{l}\text { 3. Correspondiente } \\
\text { a aspectos propios } \\
\text { del paciente } \\
\text { (F: 29) }\end{array}$} & $\begin{array}{c}\text {-No cumplió con } \\
\text { expectativas }\end{array}$ & 10 & \multirow{4}{*}{$\begin{array}{c}5 . \\
\text { Cumplimiento } \\
\text { de } \\
\text { expectativas } \\
\text { por parte del } \\
\text { paciente } \\
\text { (F: 10) }\end{array}$} & & \\
\hline & & $\begin{array}{l}\text {-Decisión } \\
\text { personal }\end{array}$ & 9 & & & \\
\hline & & $\begin{array}{l}\text {-Desmotivación } \\
\text { del paciente }\end{array}$ & 8 & & & \\
\hline & & $\begin{array}{c}\text {-Características } \\
\text { personales }\end{array}$ & 2 & & & \\
\hline Total & & $F: 108$ & & & $F: 17$ & \\
\hline
\end{tabular}

Fuente: Elaboración propia 
Según el análisis de los datos (ver Tabla 2), las razones para la terminación de la psicoterapia se agrupan en dos direcciones: en las filas, se diferencian las que tienden a explicar la finalización de la terapia más por atribuciones externas ( $F=73$ ) que por razones propias $(F=29)$. Las columnas consideran el cumplimiento o no de objetivos y tareas de la psicoterapia $(F=17)$ y, por otro lado, la terminación prematura $(F=108)$. Conforme a estos resultados, las psicoterapias tienden a terminar en mayor medida antes de realizar un cierre o reportar el cumplimiento de objetivos o tareas, y esto generalmente se da por factores de tipo institucional, socioeconómico y/o personal del paciente.

Respecto a la influencia de los factores socioeconómicos o institucionales en las decisiones de terminar la terapia, $44 \%$ de los pacientes respondieron positivamente; de los cuales, $25 \%$ refirieron dificultades económicas y $23 \%$ problemas laborales o académicos. Además, 40 \% señalaron la influencia de algún elemento asociado a la institución prestadora de servicios de salud: ubicación (17\%), disponibilidad de citas (16\%) o autorizaciones de las entidades aseguradoras (4\%). No se reportaron aspectos sociales o de orden público.

Sumado a lo anterior, los pacientes señalaron algunos factores relacionados con la psicoterapia que influyeron en su permanencia en el espacio clínico como: su motivación para estar en terapia (87\%), valoración de las acciones realizadas por el terapeuta (82\%), calidad del vínculo terapéutico (90\%) y satisfacción con el servicio (90 \%-94\%). Llama la atención que el porcentaje de aprobación de la percepción de los pacientes del progreso en terapia fue menor que el de las demás variables indagadas (63\%) (ver Tabla 3 ).

Tabla 3. Valoración de dimensiones asociadas a la psicoterapia

\begin{tabular}{ccccc}
\hline $\begin{array}{c}\text { Dimensiones asociadas a la } \\
\text { psicoterapia }\end{array}$ & Bajo & Medio & Alto & No informa \\
\hline Motivación para la terapia & 6 & 7 & 87 & \\
\hline Acciones del terapeuta & 5 & 12 & 82 & 1 \\
\hline Vínculo Terapéutico & 6 & 4 & 90 & \\
\hline $\begin{array}{c}\text { Percepción de Progreso en } \\
\text { terapia }\end{array}$ & 15 & 17 & 63 & 5 \\
\hline $\begin{array}{c}\text { Recomendaría la psicoterapia } \\
\text { Satisfacción con la terapia }\end{array}$ & 1 & 7 & 90 & 2 \\
\hline
\end{tabular}

Nota: Niveles de valoración= Bajo: Respuesta en 1 y 2, Medio: Respuesta en 3, y Alto: Respuesta en 4 y 5. Fuente: Elaboración propia

Por otro lado, a partir del análisis de regresión, las psicoterapias con término prematuro atribuido a elementos relacionados con la institución, el sistema de salud o el terapeuta ( $F$ : 39) se asociaron significativamente con pacientes que tenían bajos niveles de formación y con motivos de consulta relacionados con problemáticas de la cotidianidad, lo cual explica el $20 \%$ de la variable dependiente (X2: 9.995, Gl:2, p.0.007). Las razones para la terminación relacionadas con problemas socioeconómicos se asociaron con un bajo número de sesiones, motivo de consulta referido a problemáticas de la cotidianidad y a pacientes de menos edad, lo cual, explica el $20 \%$ de la terminación prematura de las psicoterapias ( $X^{2}:$ 16.897, Gl:3, p.0.001). 
Finalmente, los procesos que terminaron por decisión del paciente ( $F$ : 29) se asociaron con pacientes de mayor estrato económico y que reportaban bajos niveles de valoración de las acciones terapéuticas, lo cual, explica el $16 \%$ de dicha variable ( $X^{2}$ : 12.484, Gl:2, p.0.002)

En el caso de las terapias con cumplimiento de objetivos o tareas terapéuticas, se presentaron dos situaciones: cuando la terminación fue propuesta por el terapeuta, la única variable del análisis que se asoció en un $11 \%$ fue un mayor número de sesiones asistidas ( $X^{2}:$ 4.828, Gl:2, p.0.028); cuando el final es determinado por los pacientes, se asoció en un $26 \%$ con ser mujer y tener una valoración alta del vínculo terapéutico ( $X^{2}: 13.774, \mathrm{Gl}: 2$, p.0.002). Finalmente, las terapias con cumplimiento de objetivos o tareas terapéuticas en general se asocian en un $20 \%$ con ser mujer y con una valoración alta de las acciones terapéuticas (X²:13.356, Gl:2, p.0.001).

Estos resultados se observan de manera integrada en la Tabla 4.

Tabla 4. Variables asociadas al tipo de término de terapia

\begin{tabular}{|c|c|c|}
\hline $\begin{array}{c}\text { Tipos de } \\
\text { término en la } \\
\text { psicoterapia }\end{array}$ & Término prematuro & $\begin{array}{l}\text { Cumplimiento de objetivos } \\
\text { o tareas }\end{array}$ \\
\hline \multirow{2}{*}{$\begin{array}{c}\text { Atribución } \\
\text { externa al } \\
\text { paciente }\end{array}$} & $\begin{array}{l}\text { Aspectos institucionales: } \\
\text { 1) Baja formación de los pacientes y } \\
\text { 2) motivo de consulta relacionado con } \\
\text { problemáticas de la cotidianidad o } \\
\text { relacionales } \\
\text { 20\% de la varianza (X2: } 9.995, \mathrm{Gl}: 2, \text { p.0.007) }\end{array}$ & \multirow{2}{*}{$\begin{array}{l}\text { Terminar la psicoterapia por } \\
\text { parte del terapeuta: } \\
\text { 1) Alto número de sesiones } \\
\text { 11\% de la varianza ( } X^{2} \text { : } \\
4.828, G l: 2, \text { p.0.028) }\end{array}$} \\
\hline & $\begin{array}{l}\text { Aspectos socioeconómicos: } \\
\text { 1) Bajo número de sesiones, 2) Motivo de } \\
\text { consulta relacionado con problemáticas de } \\
\text { la cotidianidad o relacionales y 3) Menos } \\
\text { edad } \\
\text { 20\% de la varianza (X2: } 16.897, \text { Gl:3, p.0.001) }\end{array}$ & \\
\hline $\begin{array}{c}\text { Atribución } \\
\text { interna del } \\
\text { paciente }\end{array}$ & $\begin{array}{l}\text { Correspondiente a aspectos internos del } \\
\text { paciente: } \\
\text { 1) Mayor estrato económico y } 2) \\
\text { Valoración baja de acciones ter- } \\
\text { apéuticas } \\
16 \% \text { de la varianza }\left(X^{2}: 12.484, G l: 2, p .0 .002\right)\end{array}$ & $\begin{array}{l}\text { Cumplimiento de } \\
\text { expectativas por parte del } \\
\text { paciente: } \\
\text { 1) Ser Mujer y 2) Valoración } \\
\text { alta de vínculo terapéutico } \\
\text { 26\% de la varianza ( } X^{2} \text { : } \\
\text { 13.774, Gl:2, p.0.002) }\end{array}$ \\
\hline General & & $\begin{array}{l}\text { Cumplimiento de } \\
\text { expectativas o tareas: } \\
\text { 1) Ser Mujer y } 2) \\
\text { Valoración alta de acciones } \\
\text { terapéuticas } \\
\text { 20\% de la varianza } \\
\left(X^{2}: 13.356, \text { Gl:2, p.0.001) }\right.\end{array}$ \\
\hline
\end{tabular}

Nota: $\mathrm{X}^{2}=$ Coeficiente de correlación, $\mathrm{Gl}=\mathrm{Grados}$ de libertad, $\mathrm{p} .=$ Valor $\mathrm{p}$-Significancia Fuente: Elaboración propia

\section{Cualitativos}

Según los participantes, la finalización del proceso psicoterapéutico está relacionada con tres elementos fundamentales: 1) el incumplimiento de expectativas de la terapia, 2) los factores institucionales (aspectos externos) y, 3) el logro de objetivos (Ver Gráfico 1). 


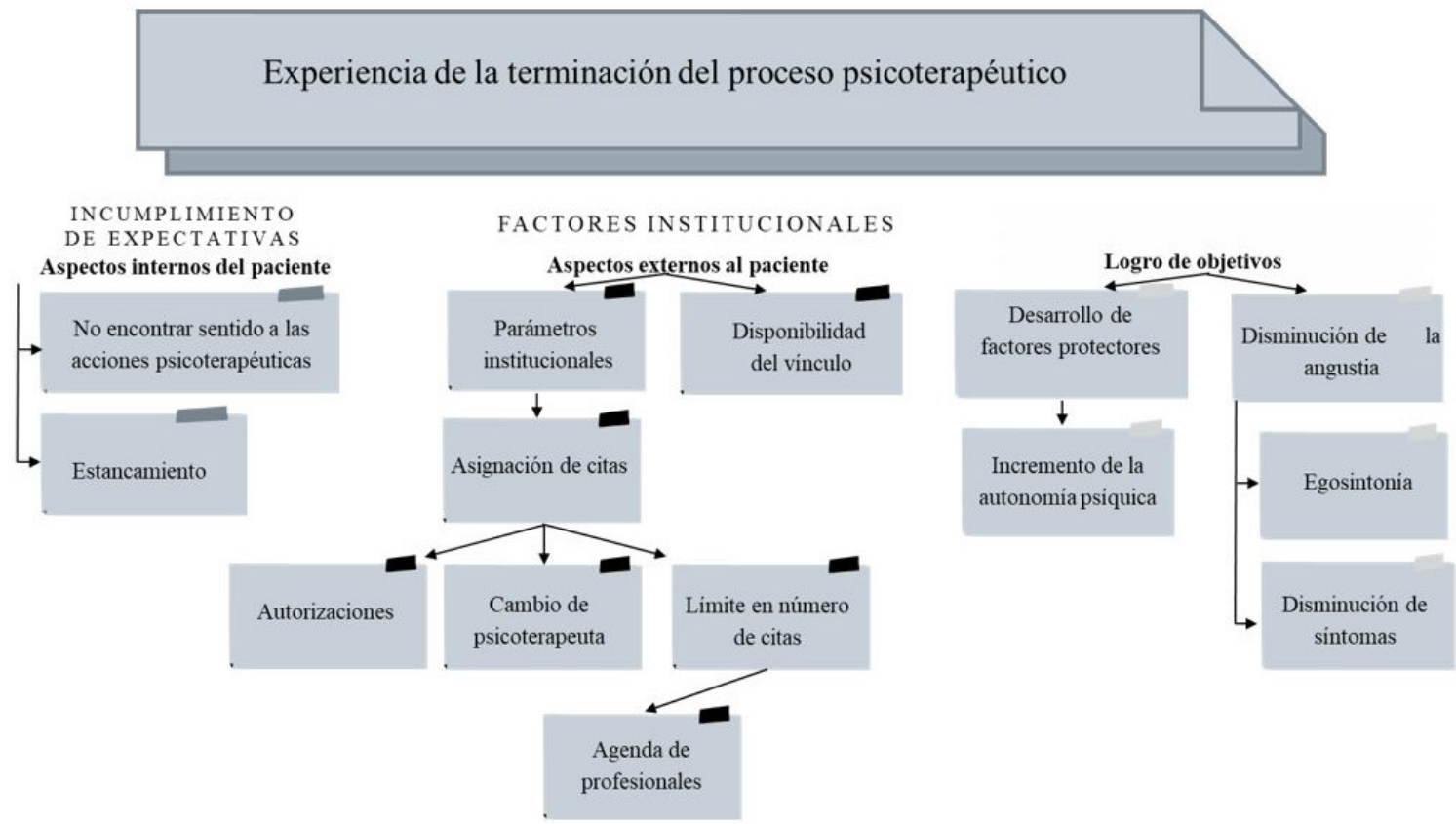

Gráfica 1. Experiencia de la terminación del proceso psicoterapéutico

Incumplimiento de expectativas (U6, U7, U8, U17, U18, U19, C4, C13).

Esta categoría se refiere a los pacientes que no encontraron sentido a lo que se hacía en el proceso (baja valoración de las acciones) o percibían estancamiento en la terapia, lo cual, constituye una de las razones centrales para dejar de asistir. En esta dirección, dos pacientes entrevistados expresan:

"Como que sentía que las preguntas me las estuviera haciendo yo misma, y yo decía no, pues para eso me hago yo las preguntas en la casa" (U1).

"Yo sentí que ya llegamos a un punto donde ella [la psicoterapeuta] no estaba aportando" (C13).

Algunos participantes tenían la expectativa de que el terapeuta realizara intervenciones más directivas o incluyera ejercicios fuera de la consulta, tal como lo menciona uno de ellos: "Nunca me ponía como: ¡bueno! haga estos ejercicios, o haga estos, como algo más dinámico" (U19). Dichas expectativas se asocian con la idea de celeridad del proceso o el logro rápido de los objetivos.

Por otro lado, algunos pacientes se sintonizaron con el tipo de intervención ofrecida, pero las condiciones del encuadre institucional no cumplieron con sus expectativas. Esto se ejemplifica en la afirmación de uno de ellos: "Lo que yo quería era que continuara pues porque siento que fueron muy pocas citas, pero igual si me ayudó mucho" (U6).

Factores institucionales (U1, U6, U8, U9, U11, U17, U18, U19, C5, C8, C9, C12, C14).

Algunos aspectos institucionales causaron desinterés en los participantes y afectaron la continuidad de los procesos; entre los que mencionaron asuntos administrativos asociados al sistema de salud como las dificultades para conseguir 
Algunos participantes señalaron que su proceso psicoterapéutico terminó debido a la consecución de logros, evidenciados en la resolución de los focos de trabajo dispuestos en el proceso, o bien, en la sensación de estar más preparados para afrontar las vicisitudes. autorizaciones y las limitaciones en el número de sesiones. Al respecto varios entrevistados reportaron:

"Yo no la fui a autorizar, porque ella me las daba aquí y yo tenía que irlas a autorizar [...], entonces yo ya no volvi" (C9).

"Yo cumplí todas las sesiones y la doctora me remitió a otras 5 sesiones, cuando yo fui a la EPS a que me las autorizaran, me dijeron que, pues, que no me las autorizaba" (C12).

Asimismo, elementos relacionados con la institución y/o el terapeuta, como la disponibilidad horaria y rotación del personal, afectaron la continuación de las terapias. Estas barreras están relacionadas con aspectos operativos como las vacaciones o actividades institucionales y tienen repercusión en la idea de disponibilidad del vínculo que tiene el consultante con el terapeuta y la institución. Así se percibe en las siguientes afirmaciones:

El final fue que nos habíamos quedado de ver para la próxima consulta y a la semana siguiente me llamó a cancelar porque estaban cancelando todas las consultas por un problema que tenían en el centro y ahí se acabó el semestre (U17).

A mí me tocó en una ocasión esperar casi 3 meses para una cita, eso fue muy maluco (C5).

Cambiar de terapeuta eso lo desestabiliza a uno (U7).

Cuando ella renunció, yo seguí la terapia particular [con ella] para no volver a empezar (C20).

Aun así, es importante resaltar que algunos entrevistados mencionaron la idea de regresar a la psicoterapia en la institución como una posibilidad.

Logro de objetivos (U4, U6, U7, U17, U19, C6, C9, C11, C15, C19, C20).

Algunos participantes señalaron que su proceso psicoterapéutico terminó debido a la consecución de logros, evidenciados en la resolución de los focos de trabajo dispuestos en el proceso, o bien, en la sensación de estar más preparados para afrontar las vicisitudes. Al respecto, uno de ellos señaló:

El proceso terminó porque [...] el psicólogo me dijo que ya me iba, me daba de alta porque[...] ya me había dado cuenta; primero ya me escuchaba y me daba cuenta obviamente, ya sabía cuáles eran mis factores protectores obviamente, pues como mis salvavidas cuando estaba en una situación de esas [...], entonces decidimos que [...] ya realmente tenía las herramientas adecuadas para seguir enfrentando [...] las situaciones que se presentaran (C20).

Uno de los referentes de logro más mencionado fue la reducción de la angustia inicial o la percepción de una mejoría a nivel sintomático. Cuando estos indicadores eran observados por el consultante y el psicoterapeuta se realizaba un cierre de la psicoterapia por "alta", tal como se aprecia en el siguiente comentario: 
Se identificaron logros asociados con la autonomía de los consultantes, que implicaron mayor conciencia sobre los temas problemáticos y la adopción de una postura de responsabilización frente a lo identificado, que, a su vez, abrió la posibilidad de realizar el cierre.
Fue como de común acuerdo los dos, porque ella me dijo: ve yo ya te veo cómo te quería ver, calmado, tranquilo, cero angustiado y te veo así, ¿te sentís así o seguimos? y yo le dije: doctora yo estoy muy tranquilo, yo ya estoy muy tranquilo, lo que pasó pues pasó" (C11).

También se identificaron logros asociados con la autonomía de los consultantes, que implicaron mayor conciencia sobre los temas problemáticos y la adopción de una postura de responsabilización frente a lo identificado, que, a su vez, abrió la posibilidad de realizar el cierre. En este sentido, dos participantes expresaron:

El descubrir que la dificultad no estaba en mi hija... en que estaba en mí, entonces yo decía: jay yo!, ¿cómo así, yo?, yo la mamá... las mamás nunca tenemos culpa de nada, pero ahí me di cuenta que sí, que las mamás tenemos mucha... no digamos culpa, pero si tenemos mucha responsabilidad (U07).

Y ahí fue donde yo mismo me empecé a mirar y claro el problema estaba en que yo reaccionaba siempre de la misma manera porque no tenía esa capacidad de hablar o de pensar claramente (U01).

\section{Elementos terapéuticos y extra terapéuticos que influyen en la terminación.}

Se identificaron aspectos externos e internos de los pacientes relacionados con el tipo de terminación de terapia. En los aspectos externos se destacan las dificultades para el desplazamiento, los trámites institucionales, las dificultades económicas y la falta de tiempo. Aunque algunos de estos aspectos habían estado presentes durante todo el proceso, adquirieron mayor relevancia al entrar en contacto con elementos internos como el sentimiento de estancamiento o la valoración de las acciones terapéuticas (Ver Gráfica 2).

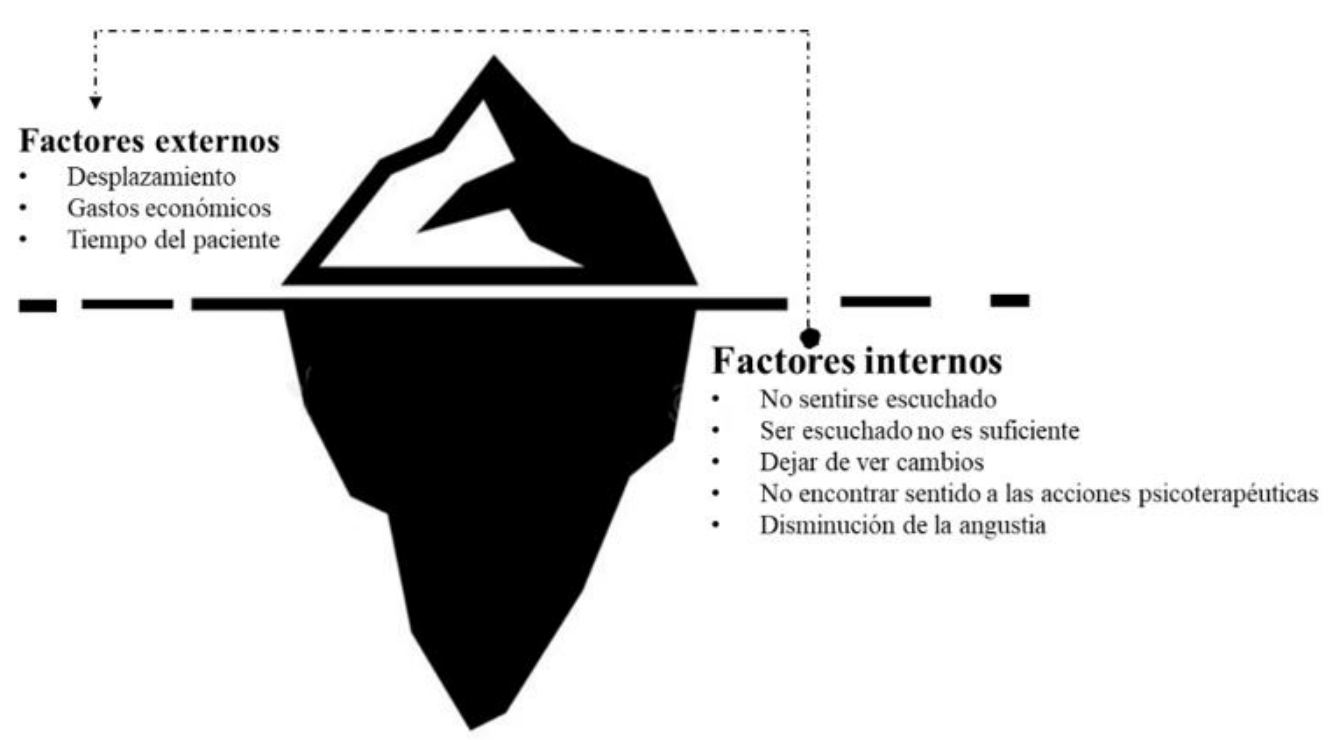

Gráfica 2. Elementos terapéuticos y extra-terapéuticos relacionados con la terminación 
Pág 214

En ambos niveles de análisis, se encontraron hallazgos similares respecto a los factores que influyen en la terminación prematura de los procesos: elementos institucionales y del sistema de salud toman relevancia al articularse con aspectos propios del paciente (internos y contextuales), que pueden estar relacionados con una valoración negativa de las acciones terapéuticas y el cumplimiento de las expectativas de la terapia.
De acuerdo con los comentarios de los participantes, se observa la confluencia de situaciones internas y/o externas a la terapia que se suman para desencadenar en un tipo de cierre específico. Con relación a esto un paciente señala: “Yo venir desde tan lejos [...] y no me daba la forma de venir, pero las dos últimas citas si fue porque ya dije: no, yo me estoy estancando" (C13).

Entre los elementos de la terapia que más influyeron en la decisión de los pacientes de terminar se incluyeron aspectos vinculares (p. ej. ser escuchado), asociados con la percepción del progreso terapéutico (p. ej. dejar de ver cambios o disminución de angustia) y con la comprensión de las acciones del terapeuta (p. ej. encontrar sentido a las intervenciones).

En ambos niveles de análisis, se encontraron hallazgos similares respecto a los factores que influyen en la terminación prematura de los procesos: elementos institucionales y del sistema de salud toman relevancia al articularse con aspectos propios del paciente (internos y contextuales), que pueden estar relacionados con una valoración negativa de las acciones terapéuticas y el cumplimiento de las expectativas de la terapia.

Por otra parte, en la terminación de terapia con cumplimiento de objetivos se encontraron elementos internos como la valoración positiva del vínculo terapéutico y la reducción de los síntomas de angustia y malestar y, elementos externos, como la decisión de cierre del proceso por parte del terapeuta, que generalmente se acompañaban de una valoración positiva de las acciones del terapeuta por parte de los pacientes.

En las terapias que finalizan con cierre por parte del terapeuta, los análisis a nivel cualitativo muestran que los participantes atribuyen esta condición en mayor medida al cumplimiento de objetivos y a su mejoría sintomática, mientras que en los análisis estadísticos también se relacionan con el alto número de sesiones.

\section{Discusión}

En la presente investigación se identificaron tres dimensiones asociadas con la terminación de los procesos psicoterapéuticos, desde la perspectivas de los pacientes: el cumplimiento o no de los objetivos, el tipo de atribución causal del paciente (interna o externa) y la persona que decide finalizar.

Respecto a la primera dimensión, se aprecia una tendencia a que las terapias se terminen antes de cumplir con los objetivos de los participantes o de manera prematura; lo que concuerda con estudios que indican que el porcentaje de pacientes que interrumpe el proceso supera el porcentaje de pacientes dados de alta (De la Parra et al., 2018). En términos generales, los objetivos son las metas hacia las que se dirige la terapia, y el grado de acuerdo y valoración de estos por parte del terapeuta y el paciente son característicos de una fuerte alianza terapéutica; y son elementos que se asocian a la terminación de las terapias (Horvath \& Luborsky, 1993). Entre los factores asociados a la terminación prematura también surgen algunas condiciones generadas por la institución y aspectos socioeconómicos relacionados con el paciente, como lo han reportado otros estudios (Westmacott et al., 2010). En esta medida, los resultados de esta investigación generan interrogantes respecto al proceso de construcción de los objetivos terapéuticos y focos de trabajo, el modo cómo son acordados con el paciente, hasta qué punto tienen en cuenta sus expectativas y posibilidades personales, y las consideraciones institucionales en el tratamiento. 
Pág 215

Respecto a los elementos de atribución interna del paciente que influyen en la terminación prematura, se encontró que una baja valoración de las acciones psicoterapéuticas es uno de los más recurrentes. Algunos autores asocian las expectativas de las personas que consultan en psicoterapia con las creencias que tienen frente al rol del psicólogo y el espacio terapéutico (Santibáñez et al. 2008).
Con relación a la atribución causal del paciente, se observa un equilibrio relativo en las razones para terminar la psicoterapia que los pacientes adjudican a la institución o sistema de salud (externas - 39\%), tales como la ubicación del centro de atención, el cambio de terapeuta, finalización del plan de citas autorizado, limitaciones horarias, dificultades con el terapeuta, e información sobre la terapia; o a sí mismos (externas - 34\% o internas 29\%), externas como el tiempo del que dispone el paciente, dificultades económicas, problemas familiares y condiciones de salud, o internas como incumplimiento de expectativas, decisión personal, desmotivación y características personales del paciente.

A nivel institucional, aspectos como la rotación de los profesionales y la continuidad de las sesiones influyen en el logro de los objetivos terapéuticos. En este sentido, algunos autores consideran que la discontinuidad en la relación paciente-terapeuta deriva en una vivencia de pérdida que tiene efecto sobre el vínculo y la motivación para la psicoterapia (Rondón et al., 2009). Se destaca, por tanto, la importancia de la continuidad de los procesos y el establecimiento de un vínculo institucional que permita sobrellevar los cambios que se puedan presentar con los profesionales tratantes.

De acuerdo con los resultados de esta investigación, una menor formación académica de los pacientes se relaciona con una terminación de terapia atribuida a factores institucionales. En esta dirección, otros estudios han señalado que aspectos socioeconómicos de los pacientes, incluido el bajo nivel de formación, pueden asociarse con mayores tasas de abandono del tratamiento psicoterapéutico y la asistencia a pocas sesiones (Swift \& Greenberg, 2012).

Algunos elementos contextuales reportados por los pacientes, como las dificultades socioeconómicas, se relacionan con las razones para terminar los procesos psicoterapéuticos de manera prematura. Esto suma evidencia al hallazgo de otras investigaciones que han reportado una conexión entre los ingresos económicos o la angustia financiera autoinformada y la poca adherencia al tratamiento o, incluso, una menor percepción de ganancias en el tratamiento (Thompson, Goldberg, \& Nielsen, 2018).

Otro aspecto relacionado con la terminación temprana de la psicoterapia se refiere al motivo de consulta, cuando este hace referencia a problemáticas de la cotidianidad y/o relacionales y no a un cuadro psicopatológico específico; lo que se puede asociar con la idea extendida de que este tipo de problemáticas son "menos graves", y pueden ser manejadas de otra manera o se sitúa la atención de la salud mental en un segundo plano.

Respecto a los elementos de atribución interna del paciente que influyen en la terminación prematura, se encontró que una baja valoración de las acciones psicoterapéuticas es uno de los más recurrentes. Algunos autores asocian las expectativas de las personas que consultan en psicoterapia con las creencias que tienen frente al rol del psicólogo y el espacio terapéutico (Santibáñez et al. 2008). En esta medida, es importante que el método utilizado por el psicoterapeuta esté sintonizado con el marco de referencia del paciente y este, a su vez, tenga la capacidad de asociar las acciones realizadas durante la sesión clínica a la sensación de mejoría o bienestar. Esta condición resalta la importancia de llegar a un acuerdo, no solo en los objetivos, sino también en las acciones que se implementan durante el tratamiento (Andrade, 2005). En este sentido, diversos autores han señalado las principales acciones terapéuticas relacionadas con la permanencia en el proceso psicoterapéutico: la relación 
La terminación del proceso por decisión unilateral del paciente, en el que este percibe cumplimiento de objetivos, se presentó en mayor medida en mujeres y en aquellos con una valoración alta del vínculo terapéutico. En esta dirección, múltiples estudios adjudican a la relación paciente-terapeuta una influencia importante en los procesos de cambio psicoterapéuticos (Safran \& Muran, 2005; Krause et al., 2006). de colaboración (Bordin, 1979), de afecto (Martin, Garske, \& Davis, 2000) y de negociación interpersonal. Estas acciones vehiculizan una experiencia de implicación de parte del psicoterapeuta, que derivan eventualmente en resolución de conflictos y la disminución del malestar subjetivo (Manubens, Roussos, Olivera, \& Gómez, 2018).

Cabe mencionar que la motivación de los pacientes frente a la permanencia en el proceso se relaciona también con la capacidad del psicoterapeuta para identificar las expectativas reales del paciente y, a partir de ellas, motivarlo a permanecer hasta el final (Moreno et al. 2012). Este proceso de contacto y cercanía con las expectativas del paciente es también un facilitador del vínculo terapéutico (Sauer, 2004).

En la experiencia de los participantes de la presente investigación la sensación de estancamiento es otra razón para abandonar el proceso. En esta línea, diversos estudios han indagado sobre elementos o momentos desfavorecedores del avance de los procesos terapéuticos como las resistencias de los pacientes, la reacción terapéutica negativa, la reactancia, refusal y las recaídas (Herrera et al., 2009), el impasse (Fossa, 2012) o el estancamiento (Krause, Echávarri, Ramírez, Valdés, \& Vilches, 2007). Estos momentos promueven el debilitamiento del vínculo terapéutico, el estancamiento del proceso y, en muchas ocasiones, suponen la terminación de este (Fossa, 2012). De ahí, la importancia de identificar las fases de estancamiento de las terapias para generar estrategias de reformulación de acciones y metas e identificación de los progresos terapéuticos con el paciente, con el fin de evitar un abandono por desconexión y facilitar el logro de los objetivos planteados (Areas et al., 2018; Laraway, 2015; Swift \& Greenberg, 2012).

Por otro lado, en la mayoría de los procesos psicoterapéuticos en los que los pacientes reportaron el cumplimiento de los objetivos, la decisión de terminar la terapia es tomada por ellos de manera unilateral. Al respecto, algunos autores plantean que durante el proceso es posible que el paciente informe al terapeuta sobre su mejoría o reducción sintomática, aunque no se hayan cumplido totalmente los objetivos terapéuticos acordados, lo que puede darle un significado de logro y no de fracaso a la terminación del proceso (Porcel, 2005; Swift, Callahan \& Levine, 2009). 0 que la percepción de mejoría frente al motivo de consulta (reducción sintomática) y la sensación de poder afrontar las angustias, es suficiente para que los pacientes finalicen la psicoterapia, aún sin tener en cuenta la opinión del profesional (Rondón et al., 2009). La terminación del proceso por decisión unilateral del paciente, en el que este percibe cumplimiento de objetivos, se presentó en mayor medida en mujeres y en aquellos con una valoración alta del vínculo terapéutico. En esta dirección, múltiples estudios adjudican a la relación paciente-terapeuta una influencia importante en los procesos de cambio psicoterapéuticos (Safran \& Muran, 2005; Krause et al., 2006).

Aunque en este estudio se diferencian los factores contextuales, institucionales y del paciente (internos y externos) que pueden influir en la terminación de las terapias, es importante recordar que dichos elementos pueden tener un peso complementario en esta decisión. En esta medida, el final de la terapia generalmente se relaciona con una suma de elementos que es necesario monitorear continuamente.

Según los hallazgos pocos procesos presentaron un cierre propuesto por el psicoterapeuta y cumplimiento de objetivos, y a su vez, la de terminación por cumplimiento de objetivos solo se asoció con la asistencia de los pacientes a un mayor número de sesiones. Este hecho puede relacionarse con el tipo de formación clínica predominante, que tiende a estar influida por enfoques de larga duración o encuadres 
particulares, en los cuales se considera la posibilidad de trabajar los objetivos de manera ilimitada.

En función de mejorar la continuidad de los procesos psicoterapéuticos a nivel institucional, los resultados expuestos invitan a pensar en tres elementos: 1) flexibilizar los requerimientos administrativos y logísticos (ubicación, horario de las citas, autorizaciones, etc.); 2) considerar dentro del encuadre algunas barreras o limitaciones temporales del proceso, como la rotación de los practicantes y los profesionales, y generar espacios que permitan promover una alianza de trabajo y un seguimiento de las intervenciones a nivel institucional y no solo individual; y 3) incluir, en los modelos de atención institucional, citas previas de indicación terapéutica, que permitan identificar si el paciente tiene las condiciones psicológicas para hacer parte de un proceso de este tipo, las características de su problemática, las expectativas referidas al tratamiento y las condiciones del contexto que pueden afectar el proceso. Estos aspectos permitirían planear mejor las intervenciones desde una lógica institucional y reducir el riesgo de terminación prematura de los procesos.

Con relación a los psicoterapeutas, los resultados señalan la importancia de incluir en la formación clínica elementos relacionados con el cierre de los procesos, los marcadores de riesgo de terminación prematura y las características de las psicoterapias institucionales; de evaluar constantemente las condiciones y expectativas de los pacientes dentro de la formulación y planeación de los casos, y apoyarlos en la identificación de los progresos terapéuticos; y de considerar la calidad del vínculo terapéutico y los niveles de aceptación, acuerdo y comprensión de las acciones que se realizan en terapia, no solo para que los pacientes se adhieran al proceso, sino para el cumplimiento de los objetivos.

Como limitaciones del presente estudio, se identificó la dificultad de abordar en las entrevistas el tema de la terminación de la terapia como fenómeno de estudio, dado que la deseabilidad social puede llevar a que los consultantes omitan información relevante. Además, la relativa homogeneidad de las instituciones participantes puede generar sesgos respecto a otro tipo de atenciones psicoterapéuticas frecuentes como la ofrecida en contextos particulares. Para próximos estudios se considera fundamental incluir la perspectiva de los psicoterapeutas, para tener una comprensión más completa de lo ocurrido en la fase final de los tratamientos.

La presente investigación permite ver un panorama diferencial de los factores por los cuales las psicoterapias llegan a su final, las dimensiones que los caracterizan y los múltiples desafíos de los servicios de psicoterapia para mantener la adherencia y concluir satisfactoriamente las intervenciones; lo que constituye un insumo importante para repensar desde una perspectiva clínica y contextual las lógicas de planeación y cierre de las terapias, identificar los riesgos de abandono y considerar los factores internos y externos del paciente que inciden y actúan de manera complementaria en los procesos psicoterapéuticos.

\section{Agradecimientos}

Este estudio recibió apoyo de la Dirección de Investigación e Innovación de la Universidad CES (proyecto número INV.032018.006), la Universidad de San Buenaventura, Medellín (proyecto número M3850) y el Fondo de Innovación para la Competitividad (FIC) del Ministerio de Economía, Fomento y Turismo, a través de la Iniciativa Científica Milenio (Proyecto IS130005). 


\section{Referencias}

Andrade, N. (2005). La alianza terapéutica. Revista Clínica y Salud, 16(1), 9-29. https:// www.redalyc.org/pdf/1806/180616109001.pdf

Areas, M., Roussos, A., Hirsch, H., Hirsch, P., Becerra, P., \& Gómez Penedo, J. M. (2018). Evaluación de un dispositivo de investigación orientada por la práctica para el desarrollo de un sistema de feedback en psicoterapia. Revista Argentina de Clínica Psicológica, 27(2), 229-249. https://dialnet.unirioja.es/servlet/articulo? codigo $=6577689$

Baekeland, F., \& Lundwall, L. (1975). Dropping out of treatment: A critical review. Psychological Bulletin, 82(5), 738-783. https://doi.org/10.1037/h0077132

Barrett, M. S., Chua, W.-J., Crits-Christoph, P., Gibbons, M. B., \& Thompson, D. (2008). Early withdrawal from mental health treatment: Implications for psychotherapy practice. Psychotherapy: Theory, Research, Practice, Training, 45(2), 247-267. https://doi.org/10.1037/0033-3204.45.2.247

Berghofer, G., Schmidl, F., Rudas, S., Steiner, E., \& Schmitz, M. (2002). Predictors of treatment discontinuity in outpatient mental health care. Social Psychiatry and Psychiatric Epidemiology, 37(6), 276-282. https://doi.org/10.1007/ s001270200020

Bordin, E. S. (1979). The generalizability of the psychoanalytic concept of the working alliance. Psychotherapy: Theory, Research \& Practice, 16(3), 252-260. https://doi. org/10.1037/h0085885

Borghi, J. H. (1968). Premature Termination of Psychotherapy and Patient-Therapist Expectations. American Journal of Psychotherapy, 22(3), 460-473. https://doi. org/10.1176/appi.psychotherapy.1968.22.3.460

Campo-Arias, A., Oviedo, H. C., \& Herazo, E. (2014). Estigma: barrera de acceso a servicios en salud mental. Revista Colombiana de Psiquiatría, 43(3), 162-167. https://doi:10.1016/i.rcp.2014.07.001

Creswell, J. W., \& Plano Clark, V. L. (2007). Designing and conducting mixed methods research. Thousand Oaks, CA: Sage Publications.

De la Parra, G., Gómez-Barris, E., Zúñiga, A. K., Dagnino, P., \& Valdés, C. (2018). Del "diván" al policlínico: Un modelo de psicoterapia para instituciones. Aprendiendo de la experiencia (empírica) Revista Argentina de Clínica Psicológica, 27(2), 182 202. https://doi.org/10.24205/03276716.2018.1057

De la Parra, G., Errázuriz, P., Gómez-Barris, E., \& Zúñiga, A. K., (2019). Propuesta para una psicoterapia efectiva en atención primaria: un modelo basado en la experiencia y la evidencia empírica. Temas de la Agenda Pública, 14(113), 1-20.

Fair, S. M., \& Bressler, J. M. (1992). Therapists-Initiated Termination of Psychotherapy. The Clinical Supervisor, 10(1), 171-189. https://doi.org/10.1300/J001v10n01 14

Fernández-Álvarez, H., \& Castonguay, L. G. (2018). Investigación orientada por la práctica: Avances en colaboraciones entre clínicos e investigadores. Introducción. Revista Argentina de Clinica Psicologica, 27(2), 107-110. https://doi.org/10.24205 I03276716.2018.1069

Fernández-Arias, I., García-Fernández, G., \& Bernaldo-de-Quirós, M. (2016). Premature termination of psychological treatment for anxiety disorders in a clinical setting. Psicothema, 28(3), 241-246. http://doi.org/10.7334/psicothema2015.201

Fossa, P. (2012). Obstáculos del proceso terapéutico: Una revisión del concepto de vínculo y sus alteraciones. Revista de Psicología GEPU, 3(1), 101 - 126.

Gersh, E., Hallford, D. J., Rice, S. M., Kazantzis, N., Gersh, H., Gersh, B., \& McCarty, C. A. (2017). Systematic review and meta-analysis of dropout rates in individual psychotherapy for generalized anxiety disorder. Journal of Anxiety Disorders, 52, 25-33. http://doi.org/10.1016/j.janxdis.2017.10.001 
González, L. M., Peñaloza, R. E., Matallana, M. A., Gil, F., Gómez-Restrepo, C., \& Landaeta, A. P. V. (2016). Factores que determinan el acceso a servicios de salud mental de la población adulta en Colombia. Revista Colombiana de Psiquiatría, 45, 89-95. https://doi:10.1016/j.rcp.2016.10.004

Hernández-Sampieri, R., \& Mendoza, C. (2018). Metodología de la investigación. Las rutas cuantitativa, cualitativa y mixta. Ciudad de México, México: Editorial Mc Graw Hill Education.

Herrera, P., Fernandez, O., Krause, M., Vilches, O., Valdés, N., \& Dagnino, P. (2009). Revisión Teórica y Metodológica de las Dificultades en Psicoterapia: Propuesta de un Modelo Ordenador. Terapia Psicológica, 27(2), 169-179.

Hopwood, C., Ambwani, S., \& Morey, L. (2007). Predicting nonmutual therapy termination with the personality assessment inventory. Psychotherapy Research, 17(6), 706-712. http://doi.org/10.1080/10503300701320637

Horvath, A. O., \& Luborsky, L. (1993). The role of the therapeutic alliance in psychotherapy. Journal of Consulting and Clinical Psychology, 61(4), 561-573. https:// doi:10.1037/0022-006x.61.4.561

Hunsley, J., Aubry, T. D., Verstervelt, C. M., \& Vito, D. (1999). Comparing therapist and client perspectives on reasons for psychotherapy termination. Psychotherapy: Theory, Research, Practice, Training, 36(4), 380-388. https://doi.org/10.1037/ $\underline{\mathrm{h} 0087802}$

Jaramillo, J. C., Espinosa, D., Ocampo, D., Arango, A., Arévalo, M., García, L., Hoyos, L., Rodriguez, V., \& Sandoval, C. (2018). Caracterización de la atención psicoterapéutica en la IPS CES Sabaneta, Colombia, 2014-2015. CES Psicología, 11(2), 97-110. http://dx.doi.org/10.21615/cesp.11.2.9

Jo, T., \& Almao, S. (2002). Abandono del tratamiento en una consulta externa del psiquiatría y psicología clínica. Revista Electrónica de portalesmédicos.com. Recuperado de http://www.portalesmedicos.com/monografias/abandono tratamiento/index.htm

Krause, M., De La Parra, G., Arístegui, R., Dagnino, P., Tomicic, A., \& Valdés, N. (2006). Indicadores genéricos de cambio en el proceso psicoterapéutico. Revista Latinoamericana de Psicología, 38(2), 299-325. https://www.redalyc.org/articulo. oa?id=80538206

Krause, M, Echávarri, O., Ramírez, I., Valdés, N., \& Vilches, O. (2007) Aprender a observar para hacer visible: Cambio y estancamiento en la terapia. En: $8^{\circ}$ Congreso Chileno de Psicoterapia: Convergencias y singularidades en psicoterapia (Viña del Mar, Chile). Trabajo no publicado.

Kleinke, C. (1998). Principios Comunes en Psicoterapia. Bilbao: Desclée De Brouwer.

Kvale, S., \& Brinkmann, S. (2009). InterViews: Learning the craft of qualitative research interviewing (2nd ed.). Sage Publications, Inc.

Lambert, M. J. (Ed.). (2012a). Bergin and Garfield's handbook of psychotherapy and behavior change (6th ed). Hoboken, New Jersey: John Wiley \& Sons.

Lambert, M. J. (Ed.). (2012b). Chapter 7. The client in psychotherapy. En Bergin and Garfield's handbook of psychotherapy and behavior change (6th ed, pp. 219-257). Hoboken, New Jersey: John Wiley \& Sons.

Laraway, C. (2015). The therapeutic alliance, ruptures, and session-by-session feedback. (Tesis Doctorado Psicología Clínica) Universidad de Oxford, Facultad de Psicología. Reino Unido, Inglaterra.

Larsen, D., Flesaker, K., \& Stege, R. (2008). Qualitative Interviewing Using Interpersonal Process Recall: Investigating Internal Experiences during Professional-Client Conversations. International Journal of Qualitative Methods, 7(1), 18-37. https:// doi:10.1177/160940690800700102 
Manubens, R., Roussos, A., Olivera, J., \& Gómez, J. (2018). Rupturas en la alianza terapéutica y su asociación con cambio y abandonos tempranos en psicoterapia. Revista de Investigación en Ciencias Sociales y Humanidades. 5(2), 143-158. http://dx.doi. org/10.30545/academo.2018.jul-dic.7

Martin, D.J., Garske, J.P., \& Davis, M.K. (2000). Relation of the therapeutic alliance without come and other variables: A meta-analytic review. Journal of Consulting and Clinical Psychology, 68(3), 438-450.

Mejía, M., \& Manjarrés, M. (2011). La investigación como estrategia pedagógica una apuesta por construir pedagogías críticas en el siglo XXI. Praxis \& Saber, 2(4), 127-177. http://doi.org/10.19053/22160159.1127

MINSALUD. (2018). Resolución 4886 de 2018: Política Nacional de Salud Mental. Bogotá: Ministerio de Salud y Protección Social. https://www.minsalud.gov.co/ Normatividad Nuevo/Resoluci\%C3\%B3n\%20No.\%204886\%20de\%202018.pdf

Moreno J. H., Rozo, M., \& Cantor, M. I. (2012). Permanencia y abandono terapéutico en un centro de servicios psicológicos. Psychologia. Avances de la Disciplina, 6(2), 23-34. http://doi.org/10.21500/19002386.1181

Monge, V. (2015). La codificación en el método de investigación de la grounded theory o teoría fundamentada. Innovaciones educativas, 17(22), 17-22. http://doi. org/10.22458/ie.v17i22.1100

Persons, J. B., Burns, D. D., \& Perloff, J. M. (1988). Predictors of dropout and outcome in cognitive therapy for depression in a private practice setting. Cognitive Therapy and Research, 12 (6), 557-575. https://doi.org/10.1007/BF01205010

Porcel, M. (2005). El abandono en las terapias psicológicas. Revista de Ciencias Sociales Aposta. (14), 1-14. http://apostadigital.com/revistav3/hemeroteca/porcel.pdf

Rancich, A. M., Pérez, M. L., Gelpi, R. J., \& Mainetti, J. A. (1999). Análisis de los principios éticos de beneficencia y no-maleficencia en los juramentos médicos, en relación con el hipocrático. Gac Med Mex, 135(3), 345-351. https://www.anmm. org. $\mathrm{mx} / \mathrm{bgmm} / 1864$ 2007/1999-135-3-345-352.pdf

Reis, B. F., \& Brown, L. G. (1999). Reducing psychotherapy dropouts: Maximizing perspective convergence in the psychotherapy dyad. Psychotherapy: Theory, Research, Practice, Training, 36(2), 123-136. https://doi.org/10.1037/h0087822

Rodríguez, C., Lorenzo, O., \& Herrera, L. (2005). Teoría y práctica del análisis de datos cualitativos. Proceso general y criterios de calidad. Revista Internacional de Ciencias Sociales y Humanidades, SOCIOTAM, 15(2), 133-154. https://www.redalyc. org/articulo.oa?id=65415209

Rojas-Bernal, L. Á., Castaño-Pérez, G. A., \& Restrepo-Bernal, D. P. (2018). Salud mental en Colombia. Un análisis crítico. Ces Medicina, 32(2), 129-140. https://doi. org/10.21615/cesmedicina.32.2.6

Rondón Benítez, Á. P., Otálora Bastidas, I. L., \& Salamanca Camargo, Y. (2009). Factors influencing therapeutic desertion of the consultants of a university counseling center. International Journal of Psychological Research, 2(2), 137-147. https:// doi:10.21500/20112084.869

Safran, J., \& Muran, C. (2005). La alianza terapéutica. Una guía para el tratamiento relacional. Bilbao: Desclée de Brouwer.

Sauer, E. (2004). Motivos de abandono de tratamiento psicológico centro médico-hospital privado de Córdoba. [Repositorio institucional] Universidad Siglo 21. Córdoba. 1-100. https://repositorio.uesiglo21.edu.ar/handle/ues21/12572

Santibáñez, P., Román, M., Lucero, C., Espinoza, A., Irribarra, D., \& Müller, P. (2008). Variables Inespecíficas en Psicoterapia. Terapia psicológica, 26(1), 89-98. https:// dx.doi.org/10.4067/S0718-48082008000100008 
Seclén-Palacín, J., \& Darras, C. (2005). Satisfacción de usuarios de los servicios de salud: Factores sociodemográficos y de accesibilidad asociados. Perú, 2000. Anales de la Facultad de Medicina, 66(2), 127-141. https://www.redalyc.org/ pdf/379/Resumenes/Resumen 37966207 1.pdf

Sharf, J., Primavera, L. H., \& Diener, M. J. (2010). Dropout and therapeutic alliance: A meta-analysis of adult individual psychotherapy. Psychotherapy: Theory, Research, Practice, Training, 47(4), 637-645. http://doi.org/10.1037/a0021175

Swift, J. K., Callahan, J., \& Levine, J. C. (2009). Using Clinically Significant Change to Identify Premature Termination. Psychotherapy, Theory, Practice, Training. 46, 328-335. https://doi.org/10.1037/a0017003

Swift, J. K., \& Greenberg, R. P. (2012). Premature discontinuation in adult psychotherapy: A meta-analysis. Journal of Consulting and Clinical Psychology, 80(4), 547-559. http://doi.org/10.1037/a0028226

Swift, J. K., \& Greenberg, R. P. (2014). A treatment by disorder meta-analysis of dropout from psychotherapy. Journal of Psychotherapy Integration, 24(3), 193-207. https://doi.org/10.1037/a0037512

Tjeltveit, A. C. (2006). To what ends? Psychotherapy goals and outcomes, the good life, and the principle of beneficence. Psychotherapy: Theory, Research, Practice, Training, 43(2), 186-200. http://doi.org/10.1037/0033-3204.43.2.186

Thompson, M. N., Goldberg, S. B., \& Nielsen, S. L. (2018). Patient financial distress and treatment outcomes in naturalistic psychotherapy. Journal of Counseling Psychology, 65(4),523-530. http://doi.org/10.1037/cou0000264

Vélez, P., \& Restrepo, D. A. (2008). El proceso de terminación en psicoterapia de tiempo limitado: aspectos clínicos y técnicos. CES Psicologia, 1(2), 58-68. https:// revistas.ces.edu.co/index.php/psicologia/article/view/80

Westmacott, R., Hunsley, J., Best, M., Rumstein-McKean, O., \& Schindler, D. (2010). Client and therapist views of contextual factors related to termination from psychotherapy: A comparison between unilateral and mutual terminators. Psychotherapy Research, 20(4), 423-435. https://doi.org/10.1080/10503301003645796

Wierzbicki, M., \& Pekarik, G. (1993). A Meta-Analysis of Psychotherapy Dropout. Professional Psychology: Research and Practice, 24(2), 190-195.

https://doi.org/10.1037/0735-7028.24.2.190

Winkler, M., Cáceres, C., Fernández, I., \& Sanhueza, J. (1989). Factores Inespecíficos de la psicoterapia y efectividad del proceso terapéutico: Una sistematización. Terapia Psicológica, (11), 34-40. 